\title{
Towards the fiber-optic Kennedy quantum receiver
}

\author{
Mikhail Elezov ${ }^{1}$, Mikhail Scherbatenko ${ }^{1}$, Denis Sych $^{1,2,3}$ and Gregory Goltsman ${ }^{1}$ \\ ${ }^{1}$ Moscow Pedagogical State University, 119992 Moscow, Russia \\ ${ }^{2}$ P.N. Lebedev Physical Institute, Russian Academy of Sciences, 119333 Moscow, Russia \\ ${ }^{3}$ JCS "QRate", Skolkovo, 143025 Moscow, Russia
}

\begin{abstract}
We consider practical aspects of using standard fiber-optic elements and superconducting nanowire single-photon detectors for the development of a practical quantum receiver based on the Kennedy scheme. Our receiver allows to discriminate two phase-modulated coherent states of light at a wavelength of 1.5 microns in continuous mode with bit rate 200 $\mathrm{Kbit} / \mathrm{s}$ and error rate about two times below the standard quantum limit.
\end{abstract}

The problem of discrimination between different non-orthogonal quantum states associated is one of the canonical problems in quantum information theory $[1,2]$. Due to the quantum uncertainty, it is impossible to discriminate without errors. The value of the error rate depends on the particular scheme of the receiver. For example, heterodyne measurements of two quantum states leads to the so-called standard quantum limit of errors, which is significantly higher than the minimum possible error rate. There are several schemes of receivers with the error rate below the standard quantum limit. However, there is a fundamental limit for the error rate, which is called the Helstrom bound [3, 4]. In order to attain the Helstrom bound, we need to have some a priori information about signal. For example, statistical distributions of single photons for different quantum states. The starting point for attaining the Helstrom bound was the Kennedy's work [5]. The Kennedy receiver has the error rate below the standard quantum limit. Various modifications of the Kennedy receiver with even lower error rate were proposed later [6-9]. All experimentally realized receivers were implemented with free-space optical components or with both free-space and fiber optic components. But there were no demonstrations of the receiver built with entirely fiber optic components. Optical fibers have many advantages over the free-space optics. They are easily scalable and reproducible due to the readily available commercial components. Also, there are highly efficient superconducting nanowire single-photon detectors with optical fiber interface [10].

In the present work, we study the features of the Kennedy receiver, which is fully implemented with standard fiber-optic components and demonstrate the error rate below the standard quantum limit. We describe the practical aspects of the operation of the fiber optic Kennedy receiver, the main sources of imperfections for signal detection, and measures to reduce their influence.

In our scheme, the optical signal is mixed with the reference signal (local oscillator), which leads to interference. The result of interference is called optical displacement. We can reveal information about the phase of the signal by measuring the interferogram. However, it is experimentally difficult to achieve spatial and temporal mode matching of two 
independent light beams. Therefore, the initial light beam is divided into two unequal parts: the signal is generated in the weak part, and the strong one serves as a local oscillator. After that, local oscillator and signal are optically mixed. An interferometric setup is usually used for all of these steps. We use the Mach-Zehnder interferometer.

In our realization of the Kennedy receiver, the main difficulties were associated with the optical fibers. There are multiple light reflections that occur at the optical connections in the fiber interferometer, which leads to degradation of the interference visibility. The MachZehnder interferometer allows to reduce the magnitude of the reflected signal. The interferometer is made with polarization maintaining fibers. In this way we can match the signal and local oscillator so that the visibility is maximal. Also, fiber optic interferometers are very sensitive to external influences, which greatly affects the stability of the resulting interferogram. Air convection, acoustic noise, thermal background radiation, etc, lead to changes in the refractive index over time in various parts of the optical path, resulting in instability of the phase difference between the interferometer arms. To reduce temperature fluctuations, all fibers of the interferometer are tightly fixed to a massive aluminum plate, which acts as a thermal bath. The whole structure is placed in a sealed metal box wrapped in a thermal insulator. After that, the stability of the interferogram increased to 100 seconds, which makes possible to carry out long measurements of the main characteristics of the Kennedy receiver. To register single photons, we used a superconducting single-photon detector with a quantum efficiency of $65 \%$ with dark counts of $100 \mathrm{~Hz}$. The receiver is capable to discriminate the binary phase-modulated signal at the speed of $200 \mathrm{kbit} / \mathrm{sec}$ with the error rate twice below the standard quantum limit.

The research has been carried out with the support of the Russian Science Foundation (project No. 1772-30036)

\section{References}

1. W. K.Wootters and W. H. Zurek, Nature (London) 299, 802 (1982).

2. D. Dieks, Phys. Lett. 92A, 271 (1982).

3. P. A. Bakut and S. S. Shchurov, Probl. Peredachi Inf. 4, 77 (1968).

4. C. W. Helstrom, Inf. Control 10, 254 (1967).

5. R. Kennedy, MIT Res. Lab. Electron. Quart. Prog. Rep. 108, 219 (1973).

6. S. Dolinar, MIT Res. Lab. Electron. Quart. Prog. Rep. 111, 115 (1973);

7. M. Sasaki and O. Hirota, Phys. Rev. A 54, 2728 (1996).

8. M. Takeoka and M. Sasaki, Phys. Rev. A 78, 022320 (2008).

9. D. Sych and G. Leuchs, Phys. Rev. Lett 117, 200501 (2016).

10. G.N. Gol'tsman, et al, Appl. Phys. Lett. 79, 705-707 (2001) 